\title{
Effects of Gibberellic Acid on Gene Expression of Menthol Biosynthesis Pathway in Peppermint (Mentha piperita L.)
}

\author{
Farid Soleymani ${ }^{1}$, Henghameh Taheri ${ }^{2}$ and Alireza Shafeinia ${ }^{2}$
}

\begin{abstract}
Peppermint is the major source of menthol rich essential oil and widely cultivated for pharmaceutical purposes. In this study, the effect of exogenous applications of gibberellic acid (GA3) was investigated in the main genes of menthol biosynthesis pathways within a $72 \mathrm{~h}$ time period using qRT-PCR. Transcript accumulation for the respective enzymes of early pathway steps relatively remained unaffected whereas expression levels of genes in later stages of oil biosynthesis, including neo-red and $m$-deh were down-regulated by GA3 treatment which is likely related to the negative feedback of rising GA levels following elicitor application in transcript level of genes involved in isoprenoid biosynthesis.
\end{abstract}

Keywords - Mentha x piperita, Menthol, qRT-PCR, Gibberellic acid.

\section{INTRODUCTION}

$\mathbf{M}^{\circ}$ ONOTERPENES belong to $\mathrm{C}_{10}$ isoprenoids that constitute the major components of the essential oils of mint family, including peppermint (Mentha piperita L.) and spearmint (Mentha spicata) [1]. Biosynthesis of monoterpens including menthol are restricted to highly specialized nonphotosynthetic secretory cells derived from the epidermal layer called peltate glandular trichomes. The major pathway for biosynthesis of menthol is conducted by eight enzymatic reactions. Menthol formed from the assembly of $\mathrm{C}_{5}$ isoperne units, namely IPP and its allylic isomer DMAPP to produce acyclic precursor geranyl diphosphate by GPPS [2]. The second specific step of the pathway is cyclization of GPP by plastidial (-)-(4S) limonene synthase and thereby establishes limonene [3]. Following hydroxylation at C3 and a sequence of four redox reactions on cyclohexanoid ring affords (-) - (1R, 3R, 4S) menthol [4]. This valuable natural product has considerable economic importance due to its multitude aromatherapy and industrial applications (5). An important menthol biosynthesis pathway side-product is menthofuran. This undesirable monoterpen is derived from C9 hydroxylation, cyclization and dehydration of (+)- pulegone by an endoplasmic reticulum- localized enzyme called menthofuran synthase. It's worth mentioning that abiotic stress, promotes the accumulation of menthofuran and pulegone metabolites during leaf expansion period [6]. Metabolic

\footnotetext{
${ }^{1}$ Student, Department of Agronomy and Plant Breeding, Ramin Agriculture \& Natural Resources University of Khouzestan, Iran. E-mail: faridsoleymani2012@gmail.com

${ }^{2}$ Assistant Professor, Department of Agronomy and Plant Breeding, Ramin Agriculture \& Natural Resources University of Khouzestan, Iran. Tel: 00986133222424, Fax: 00986133222425,

E-mail: hetaheri@alumni.ut.ac.ir, shafeinia@alumni.ut.ac.ir
}

engineering approaches have been undertaken to improve quantitative and compositional changes in commercially distilled peppermint oil [7-9].

It is worth noting that many pharmaceutical metabolites produce when plants subjected to stresses, including various elicitors or signal molecules like hormones [10, 11]. Gibberellins are recognized compounds that play an important role in the eliciting the biosynthesis of secondary metabolites in plant cells. Gibberellins are also reported to increase plant biomass and menthol content In $M$. piperita [12]. In spite of the fact that isoprenoids are a structurally and functionally diverse group of natural products, all of them are derived from two $\mathrm{C}_{5}$ units provided by cytosolic MVA and plastidial MEP pathways. Sequential elongation reactions with the addition of one, two or three IPP units lead to the biosynthesis of GPP $\left(\mathrm{C}_{10}\right)$, FPP $\left(\mathrm{C}_{15}\right)$ and GGPP $\left(\mathrm{C}_{20}\right)$ which are the starting points of downstream pathways for the production of monoterpenes, sesquiterpenes and diterpens respectively [13]. The MEP pathway provides IPP for the synthesis of monoterpenes, carotenoids, abscisic acid and gibberellin. Gibberellins are usually produced by plastidial GGPP derived from MEP pathway. In the MEP pathway, eight enzymatic reactions are used to yield GA from GGPP. Analysis of the expression of genes involved in GA biosynthesis revealed new insights into the regulation of GA concentration in Plants [14, 15].

This study was performed to evaluate the effect of timecourse exogenous application of gibberellic acid $\left(\mathrm{GA}_{3}\right)$ on transcript level alterations of genes involved in monoterpene biosynthesis under this treatment.

\section{MATERIALS AND METHODS}

\section{2-1. Plant Materials, $G A_{3}$ Treatment and Samplings}

This experiment was carried out under natural light conditions in the greenhouse of Ramin Agriculture and Natural Resources University of khouzestan (IRAN). The rhizomes of peppermints were collected from Pakanbazr Company, Esfahan, Iran. Then $10-15 \mathrm{~cm}$ rhizomes were transferred into pots. They were watered every day. Two months-old uniform plants were selected for sampling.

In order to treat the plants $50 \mathrm{mg} / \mathrm{L} \mathrm{GA}_{3}$ sprayed on the surface of the peppermint. The untreated peppermint plants (control) were sprayed with only distilled water. Leaves from the untreated (control) and treated peppermint plants were randomly sampled at 12, 24 and $72 \mathrm{~h}$ after treatment. For each sampling, 4 leaves under the second visible leaf from the apex were harvested, frozen in liquid nitrogen, stored at $-80{ }^{\circ} \mathrm{C}$ 
immediately for RNA extraction. In order to more accuracy of experiments, and separate associated control was individually considered for each treatment.

\section{2-2. RNA Extraction,cDNA Synthesis, Primer DesignandqRT-PCR Reaction}

Total RNA was isolate from mentha leaves using GeneAll® RiboEx ${ }^{\mathrm{TM}}$ kit (BioFrontier, Korea) based on manufacturer's protocol. The quality of extracted RNA was checked by $1 \%$ agarose gel electrophoresis. The first strand cDNA was synthesized from 500 ng of total RNA, using Fermentas kit (RevertAid TM First Strand cDNA Synthesis Kit) according to the manufacturer's instructions.

Primer pairs of $p r, m f s, l s, l 3 h$, iso-red, neo-red, $m$-deh and $g d s$ genes were designed with online Primer Quest software according to the cDNA sequences (pr (AY300163.1), mfs (AF346833.1), ls (EU108697.1), l3h (AF124817), iso-red (AY300162), neo-red (DQ362936), m-deh (AY288138) and gds (AF182828.1) of M. x piperita, and actin (KM044035.1) of $M$. spicata that was employed as an internal standard (table 1). To ensure the specific amplification of designed primers for these genes, PCR reaction was performed using cDNA.

The qRT-PCR was performed using HIFI SYBR ${ }^{\circledR}$ Green kit (Iran) Master mix and Step One Plus® (ABI, America) machine under the following conditions: $95^{\circ} \mathrm{C}$ for $30 \mathrm{~s}$ followed by 40 cycles $95^{\circ} \mathrm{C}$ for $15 \mathrm{~s}, 52^{\circ} \mathrm{C}$ for $20 \mathrm{~s}$ and $72^{\circ} \mathrm{C}$ for $20 \mathrm{~s}$. Relative expression levels of the genes were calculated by $\Delta \Delta \mathrm{CT}$. Relative Expression Software Tool $\left(\right.$ REST) ${ }^{\circledR}$ software [16] was used to analyses of data. This experiment was carried out with two biological and two technical repeats.

\section{RESULTS AND DISCUSSION}

\subsection{Expression of Main Genes in Menthol Biosynthetic} Pathway

To investigate whether exogenous application of $\mathrm{GA}_{3}$ at different time $(12,24$ and $72 \mathrm{~h})$ after treatment affects transcription of the main genes in menthol biosynthesis, we used qRT-PCR to evaluate the responses of the genes $g d s, l s$, $l 3 h$, iso-red, $p r, m f s$, neo-red and m-deh. Transcript levels changes of genes in plants exposed to $\mathrm{GA}_{3}$ were slightly variable. The transcript level of $g d s$ was increased 6 fold than in the untreated control at $12 \mathrm{~h}$, whereas thereafter did not significantly change. The expression level of $l s$, iso-red, $m f s$ and $p r$ genes didn't show significant changes at all mentioned times after treatment. The $13 \mathrm{~h}$ mRNA level increased whithin $12 \mathrm{~h}$ of $\mathrm{GA}_{3}$ elicitor treatment (5.2 fold higher than that in the control) while at 24 and $72 \mathrm{~h}$ expression changes were not remarkable. The transcript levels of neo-red gene (Catalysing the conversion menthone to neomenthol or isomenthone to isomenthol) decreased markedly to below the control at $12 \mathrm{~h}$ (approximately 1.4 times lower than those in the control) as well as the expression of $m$-deh (catalysing the conversion of menthone to menthol or isomenthone to neoisomenthol) notably reduced ( 3 fold lower than those in the control) during $24 \mathrm{~h}$ following $\mathrm{GA}_{3}$ application. (Figure 2).

A regulatory role of DXS (Catalyzing the first reaction of the MEP pathway) in controlling the synthesis of MEP-derived isoprenoids came from the analysis of transgenic plants in which DXS was upregulated. A distinct positive correlation between DXS transcript levels and the synthesis of plastidic isoprenoids has been reported in Arabidopsis [36] and in $M$. piperita [8]. In vegetative cannabis plants, reduction in DXS activity was parallel with the reduction in chlorophyll and carotenoid contents by $\mathrm{GA}_{3}$ application. Furthermore, the number and percentage of mono and sesquiterpens - derived MEP pathway declined in treated plants. This finding tightly has confirmed new insights into limiting the role of the MEP pathway in the synthesis of plastidic isoprenoids by exogenous application of $\mathrm{GA}_{3}$ [17]. The current study also demonstrated that transcript accumulation for the respective enzymes of early pathway steps remained unaffected under $\mathrm{GA}_{3}$ treatment. One other interesting result is elucidated that expression levels of genes in later stages of oil biosynthesis, including neo-red and $m$-deh was down-regulated by $\mathrm{GA}_{3}$ treatment. This result is consistent with finding in former study, which observed no substantial alterations in transcript levels of genes dedicated to the early steps of oil biosynthesis $(g d s, l h, l s)$ in response to $\mathrm{GA}_{3}$ in $M$. arvensis [18]. A recent study also has reported the effect of $\mathrm{GA}_{3}$ on the stimulation of trichome formation and increases in its density and diameter on $M$. arvensis eventually resulted in increase oil yield. Since the biosynthesis of gibberellins are derived from MEP pathway, Most of genes involved in the formation of bioactive GA, downregulated by applying GA. Existing evidences confirmed that GA biosynthesis is regulated by feedback control $[19,20]$. This self-regulation mechanism may interfere with biosynthesis of monoterpens and other isoprenoids derived from MEP pathway.

\section{CONCLUSIONS}

A set of genes involved in the menthol biosynthetic pathway was selected for qRT-PCR to analyze their expression in phyto-hormonal $\mathrm{GA}_{3}$ treated and control plants. On the whole, these results showed that with the few exceptions noted above, most transcript levels of genes were either unaffected or downregulated. Since exogenous application of $\mathrm{GA}_{3}$ downregulate transcript levels of several genes involved in GA biosynthesis and other isoprenoids, there is this expectance that $\mathrm{GA}_{3}$ treatment might not have a prominent role in enhancing menthol yield. However to understand the role of $\mathrm{GA}_{3}$ negative feedback mechanism in menthol biosynthesis, we need further investigation. More profound studies on genes affecting trichomes formation and development, as well as menthol production, could be the next step for a further improvement yield of this valuable therapeutic.

\section{ACKNOWLEDGMENTS}

The experiment was performed in the central laboratory of Ramin University, Khouzestan, Iran. The authors are thankful to the laboratory personals for putting all the means at our disposal.

Nomenclature: $g d s$ : Geranyl diphosphate synthase, $l s$ : Limonene synthase, l3h: Cytochrome P450 (2)-limonene-3hydroxylase, iso-red: Isopiperitenone reductase, $p r$ : Pulegone reductase, $m f s$ : Menthofuran synthases, neo-red: Neomenthol reductase, m-deh: Menthol dehydrogenase, IPP: Isopentenyl 
diphosphate, DMAPP: Dimethylallyl diphosphate, ROS: Reactive oxygen species, MVA: Mevalonic acid, MEP: methyl erythritol phosphate, DXS: 1-deoxy-D-xylulose 5-phosphate synthase, GGPP: geranyl geranyl diphosphate, FPP: Farnesyl pyrophosphate.

\section{REFERENCES}

[1] B. M. Lawrence, "Monoterpene interrelationships in the Mentha genus: a biosynthetic discussion. In: Mookherjee BD, Mussinan CJ, editors. Essential Oils. Wheaton, IL: Allured Publishing, pp. 1-81, 1981.

[2] C. C. Burke, M. R. Wildung, and R. Croteau, "Geranyl diphosphate synthase: Cloning, expression, and characterization of this prenyltransferase as a heterodimer,"Proc Natl Acad Sci USA,vol. 96. Pp, 13062-13067, 1999.

[3] W. R. Alonso, J. I. M. Rajaonarivony, J. Gershenzon and R. Croteau, "Purification of 4S-limonene synthase, a monoterpene cyclase from the glandular trichomes of peppermint (Mentha piperita) and spearmint (M. spicata)," J Biol Chem, vol. 267, pp.7582-7587, 1992.

[4] R. Croteau, and R. Gershenzon, "Genetic control of monoterpene biosynthesis in mints (Mentha:Lamiaceae),". Rec Adv Phytochem,vol. 28, pp. 193-229, 1994.

[5] B.M. Lange A. Ahkami, (2013) "Metabolic engineering of plant monoterpenes, sesquiterpenes and diterpenes - current status and future opportunities," Plant Biotechnol, vol. 11, pp. 169-196, 2013. http://dx.doi.org/10.1111/pbi.12022

[6] R. J. Clark, and R. C. Menary, "Environmental Effects on Peppermint (Mentha piperita L.). I. Effect of Daylength, Photon Flux Density, Night Temperature and Day Temperature on the Yield and Composition of Peppermint Oil," Australian Journal of Plant Physiology, ,vol. 7, pp. $685-692,1980$ http://dx.doi.org/10.1071/PP9800685

[7] S. S. Mahmoud, M. Williams and R. Croteau,"Cosuppression of limonene-3-hydroxylase in peppermint promotes accumulation of limonene in the essential oil,"Phytochemistry, vol.65, pp. 547-54, 2004. http://dx.doi.org/10.1016/j.phytochem.2004.01.005

[8] S. S. Mahmoud, and R. Croteau,"Menthofuran regulates essential oil biosynthesis in peppermint by controlling a downstream monoterpene reductase,"Proc Natl Acad Sci USA, vol. 98, pp. 8915-8920, 2001. http://dx.doi.org/10.1073/pnas.141237298

[9] F. Diemera, J. C. Caissarda, S. Moja, J. C. Chalchat and F. Jullien,"Altered monoterpene composition in transgenic mint following the introduction of 4S-limonene synthase,"Plant Physiology and Biochemistry, vol.39, pp. 603-614, 2001. http://dx.doi.org/10.1016/S0981-9428(01)01273-6

[10] J. Zhao, L. C. Davi and R. Verpoorte,"Elicitor signal transduction leading to production of plant secondary metabolites,"Biotechnol Adv, vol. 23 , pp. 283-333, 2005. http://dx.doi.org/10.1016/j.biotechadv.2005.01.003

[11] A. Ramakrishna, and G. A. Ravishankar,"Influence of abiotic stress signals on secondary metabolites in plants,"Plant Signal Behav, vol 6, pp. 1720-1731, 2011 http://dx.doi.org/10.4161/psb.6.11.17613

[12] P. Singh, and A. Mishra, "Influence of gibberellins and ethereal on growth, chlorophyll content, protein, enzyme activities and essential monoterpene oil in efficient genotype mentha spicata var. MSS-5, J. Med, Aromat," Plant sci, vol. 22, pp. 283-286, 2001.

[13] T. Ogura, K. Inoue, T. Tatsuta, T. Suzaki, K. Karata, K. Young, L. H. Su, C. A. Fierke, J. E. Jackman, C. R. H. Raetz, J. Coleman, T. Tomoyasu, and H. Matsuzawa, "Balanced biosynthesis of major membrane components through regulated degradation of the committed enzyme of lipid A biosynthesis by the AAA protease FtsH (HflB) in Escherichia coli," Molecular Microbiology, vol. 31, pp. 833-844, 1999. http://dx.doi.org/10.1046/j.1365-2958.1999.01221.x

[14] P. Hedden, and A. L. Philips, "Gibberellin metabolism: New insight revealed by the genes,"Trends Plant Sci, vol. 5, pp. 523-530, 2000. http://dx.doi.org/10.1016/S1360-1385(00)01790-8

[15] N. Olszewski, T. P. Sun and F. Gubler,"Gibberellin signaling: biosynthesis, catabolism, and response pathways," The Plant cell, vol14, pp. 61-80, 2002.

[16] M. Pfaffl, "A new mathematical for relative quantification in realtime RT-PCR," Nucleic acids Res, vol 29, pp e45, 2001. http://dx.doi.org/10.1093/nar/29.9.e45

[17] H.Mansouri, Z. Asrar, and A. Ryszard,"The response of terpenoids to exogenous gibberellic acid in Cannabis sativa $L$. at vegetative stage,"Acta Physiol Plant, vol. 33, pp.1085-1091, 2011. http://dx.doi.org/10.1007/s11738-010-0636-1

[18] S. K. Bose, R. K. Yadav, S. Mishr, R. S. Sangwa, A. K. Sing, B. Mishr, A. K. Srivastav, N. S. Sangwan,"Effects of gibberellic acid and calliterpenone on plant growth attributes, trichomes, essential oil biosynthesis and pathway gene expression in differential manner in Mentha arvensis L,"Plant Physiol Biochem, vol. 66, pp.150-158, 2013. http://dx.doi.org/10.1016/j.plaphy.2013.02.011

[19] D. N. Martin, W. M. Proebsting, T. D. Parks, W. G. Dougherty, T. Lange, M. J. Lewis, P. Gaskin and P. Hedden,"Feed-back regulation of gibberellin biosynthesis and gene expression in Pisum sativum L,"planta, vol. 200, pp. 159-166, 1996.

[20] J. J. Ross, A. K. MacKenzie-Hose, P. J. Davies, D. R. Lester, B. Twitchin and J. B. Reid,"Further evidence for feedback regulation of gibberellin biosynthesis in pea,"Physiologia Plantarum, vol. 105, pp. 532-538, 1999.

http://dx.doi.org/10.1034/j.1399-3054.1999.105319.x 
TABLE I

Sequence, Tm And Product Size Of ACT, GDS, LS, L3H, ISO-RED, PR, MFS, NEO-RED AND M-DEH Primers.

\begin{tabular}{|c|c|c|c|c|}
\hline Gene & primer & sequence $\left(5^{\prime}-3^{\prime}\right)$ & $\mathrm{TM}\left({ }^{\circ} \mathrm{C}\right)$ & $\begin{array}{c}\text { Product size } \\
\text { (bp) }\end{array}$ \\
\hline \multirow[t]{2}{*}{ act } & $\mathrm{F}$ & TCCTGAGAGGAAGTACAGTGTC & 62 & 108 \\
\hline & $\mathrm{R}$ & GACGGCCCAGATTCATCATAC & 62 & \\
\hline \multirow[t]{2}{*}{$l s$} & $\mathrm{~F}$ & TGACAGAGGTGTGGAAGAAG & 62 & 113 \\
\hline & $\mathrm{R}$ & GTACATCAACTGCGCCATC & 62 & \\
\hline \multirow[t]{2}{*}{$p r$} & $\mathrm{~F}$ & $\begin{array}{l}\text { GAAGCTGTGATCAACAACATGA } \\
\text { G }\end{array}$ & 62 & 126 \\
\hline & $\mathrm{R}$ & ACGAATTTGCTTTGGGATTAGC & 62 & \\
\hline \multirow[t]{2}{*}{$m f s$} & $\mathrm{~F}$ & TGACTGAAGCTCCTGGATTTG & 62 & 111 \\
\hline & $\mathrm{R}$ & СCTTCCCTTCCGTGTGTATATG & 62 & \\
\hline \multirow[t]{2}{*}{ Neo-red } & $\mathrm{F}$ & CAGAGGAGAAACTGGAGGAAG & 62 & 115 \\
\hline & $\mathrm{R}$ & GCTGCTTTCGACACTTTGTAG & 62 & \\
\hline \multirow[t]{2}{*}{ M-deh } & $\mathrm{F}$ & TCGGATCATAGCGCGAAAG & 62 & 112 \\
\hline & $\mathrm{R}$ & AGCACCTTCAGCTTCACTTAG & 62 & \\
\hline \multirow[t]{2}{*}{$g d s$} & $\mathrm{~F}$ & TAGGGCAGCTCCATTGATTG & 62 & 119 \\
\hline & $\mathrm{R}$ & AGAAAGGAGCATCATGTTTGTG & 62 & \\
\hline \multirow[t]{2}{*}{$L 3 h$} & $\mathrm{~F}$ & ATTTCGAGTTCGTCCCGTTC & 62 & 129 \\
\hline & $\mathrm{R}$ & TCATTCСТTCCGCCAACTTC & 62 & \\
\hline \multirow[t]{2}{*}{ Iso-red } & $\mathrm{F}$ & CGAAGAAGTACCCGAGTTTCC & 62 & 115 \\
\hline & $\mathrm{R}$ & TTCACCGGAACTTGAGCAG & 62 & \\
\hline
\end{tabular}

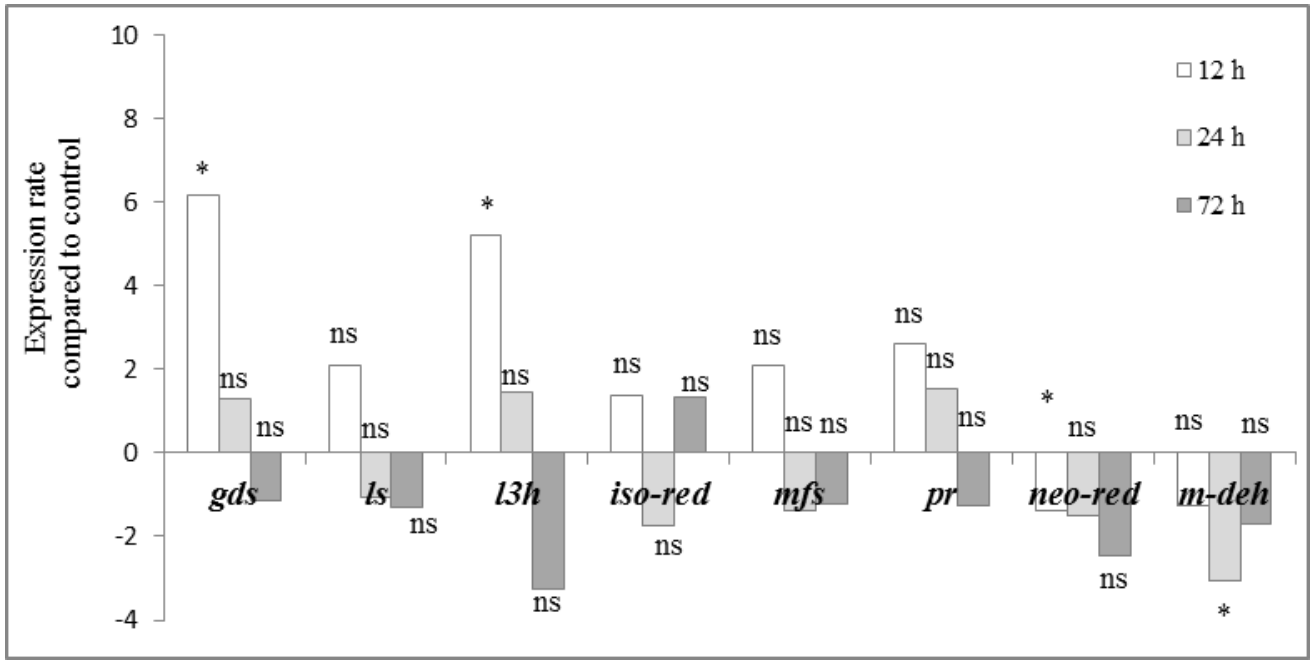

Fig. 1:, The relative expression rate of $l s, g d s, l 3 h$, iso red, $m f s, p r$, neo-red and $m$-deh genes in times after applying $\mathrm{GA}_{3}$ treatment. $*$ and "ns" indicate significant differences respectively at $(\mathrm{P}<0.05)$ and non- significant differences between treat and itself control. 